\title{
Effect of sleep deprivation on overnight bronchoconstriction in nocturnal asthma
}

\author{
J R CATTERALL, G B RHIND, I C STEWART, K F WHYTE, C M SHAPIRO, \\ N J DOUGLAS
}

From the Department of Respiratory Medicine and Rayne Laboratory, University of Edinburgh, City Hospital, Edinburgh

ABSTRACT Nocturnal cough and wheeze are common in asthma. The cause of nocturnal asthma is unknown and there is conflicting evidence on whether sleep is a factor. Twelve adult asthmatic subjects with nocturnal wheeze were studied on two occasions: on one night subjects were allowed to sleep and on the other they were kept awake all night, wakefulness being confirmed by electroencephalogram. Every patient developed bronchoconstriction overnight both on the asleep night, when peak expiratory flow (PEF) fell from a mean (SE) of 418(40) $1 \mathrm{~min}^{-1}$ at $10 \mathrm{pm}$ to $270(46) 1$ $\mathrm{min}^{-1}$ in the morning, and on the awake night (PEF $10 \mathrm{pm} \mathrm{465(43),} \mathrm{morning} 371(43) 1 \mathrm{~min}^{-1}$ ). The morning values of PEF were, however, higher $(p<0 \cdot 1)$ after the awake night and both the absolute and the percentage overnight falls in PEF were greater when the patients slept (asleep night $38 \%$ $(6 \%)$, awake night $20 \%(4 \%) ; p<0.01)$. This study suggests that sleep is an important factor in determining overnight bronchoconstriction in patients with nocturnal asthma.

Asthmatic patients often wheeze at night and show a fall in peak expiratory flow (PEF) in the early morning. ${ }^{1}$ At best this nocturnal bronchoconstriction is annoying and disrupts sleep, ${ }^{2}$ but at worst it may be life threatening as there appears to be an excess of deaths and ventilatory arrests at night in asthmatic patients. ${ }^{4-6}$

Many factors, including circadian changes in hormone secretion and diurnal variation in bronchial reactivity, have been proposed to explain the worsening of asthma at night, but the cause of nocturnal asthma is unknown. ${ }^{6}$ It is still unclear whether sleep itself is essential for the development of nocturnal wheezing. ${ }^{6}$ We have therefore kept 12 asthmatic patients who reported nocturnal wheezing awake all night to see whether this altered their nocturnal bronchoconstriction or their morning bronchial reactivity to histamine.

\section{Methods}

PATIENTS

We studied 12 asthmatic patients (nine men and three

Address for reprint requests: Dr NJ Douglas, University Department of Respiratory Medicine, City Hospital, Edinburgh EH10 5SB.

Accepted 24 February 1986 women) aged 22-58 years. All had documented nocturnal bronchoconstriction, their PEF recorded four times daily in the week before study showing an average diurnal change-(highest PEF-morning PEF)/highest PEF - of 29\% (range 10-68\%). Eight had positive skinprick test responses to at least two common allergens and seven had a family history of atopy. Six were lifelong non-smokers, four (two atopic) were ex-smokers, and two (both atopic) smoked 10-15 cigarettes a day. All patients had been attending our clinic for at least one year but none had had an acute attack of asthma in the six weeks before the study. All used $\beta_{2}$ agonist inhalers and one an anticholinergic inhaler, nine inhaled steroids, and four inhaled sodium cromoglycate. Three patients took 5-10 mg daily of oral prednisolone and five were taking oral theophyllines, which were discontinued for 24 hours before each study night.

PROCEDURE

Each patient was studied on two occasions, an "awake night" and an "asleep night" 7-14 days apart, the order of the two nights being randomly determined.

On the "asleep night," which was preceded by a 뭉 night of acclimatisation in the same conditions, each patient slept undisturbed in a quiet, darkened room. PEF was recorded in the seated position before sleep 
at $10 \mathrm{pm}$, after sleep at $7 \mathrm{am}$, and also if the patient awoke during the night asking to use an inhaler. All PEF measurements on each night were made by the same technician using the same instructions. Sleep was scored $^{8}$ from an electroencephalogram (EEG) with two frontoparietal electrodes, an electrooculogram with four electrodes above and lateral to the outer canthi, and an electromyogram with two submental electrodes.

On the "awake night" the patients played cards or board games and watched non-stimulating films; they were permitted to walk slowly around the sleep laboratory complex but were not allowed out of doors, and such mild exertion was always less than 15 minutes a night. They were denied substances containing caffeine as these are bronchodilators. ${ }^{9}$ They were supervised constantly by two members of staff, who ensured that they did not sleep; and whenever they were seated or lying wakefulness was confirmed by EEG. On the awake night PEF was recorded at hourly intervals, again with the subjects seated. At 7 am after each study night bronchial reactivity to histamine was assessed ${ }^{10}$ to see whether overnight wakefulness blunted bronchial reactivity.

Before each study the subjects performed a normal day's work and were instructed not to sleep. Venous blood was drawn at the beginning of each study; it contained very low concentrations of caffeine (less than $0.9 \mu \mathrm{g} / \mathrm{ml}$ ) in all patients and detectable theophyllines in only five patients - in each case the theophylline level was $<3 \cdot 3 \mu \mathrm{g} / \mathrm{ml}$.

All subjects gave informed consent to the study, which was approved by the hospital ethical committee. Results are quoted as means with standard errors in parentheses, and the significance of differences was assessed by Student's $t$ test for paired data or by analysis of variance and Duncan's multiple comparison test. ${ }^{11}$

\section{Results}

\section{EEG SLEEP STAGE}

On the awake night all patients remained awake throughout the night. On the asleep night the mean (SD) sleep period was 413 (14) minutes, during which there were 55(16) minutes of wakefulness, 21 (4) minutes of stage 1 sleep, 172(11) minutes of stage 2 , 43 (5) minutes of stage 3, 56(9) minutes of stage 4, and $66(10)$ minutes of rapid eye movement (REM) sleep.

\section{OVERNIGHT BRONCHOCONSTRICTION}

Although PEF at $10 \mathrm{pm}$ on the awake night $(465(43)$ $1 \mathrm{~min}^{-1}$ ) was higher than on the asleep night (418(40) $\left.1 \min ^{-1}\right)$, the difference was not significant $(0 \cdot 1>p>0 \cdot 05)$. Seven of the 12 patients did not use an inhaler on either night; three did so only on the sleep night (at 2.40, 4.00 and 5.30) and two used their inhalers on both nights (at 1.00 and 4.20 on the asleep night and at 3.00 and 6.00 respectively on the awake night). Thus no patient used his inhaler earlier on the awake night than on the asleep night. The overnight changes in PEF were compared on the basis of the 7 am values in the seven patients who did not use inhalers, and comparing the pre-inhaler PEF on the asleep night with the nearest time-matched PEF on the awake night in the other five patients.

All patients showed overnight bronchoconstriction both on the asleep and on the awake night (fig 1). The PEF fell to lower values on the asleep night (270(46) $\left.1 \mathrm{~min}^{-1}\right)$ than on the awake night (371 (43) $1 \mathrm{~min}^{-1}$; $\mathrm{p}<0.01$ ). The overnight fall in PEF was significantly greater on the asleep night than on the awake night, both in terms of absolute fall in PEF (asleep night 148 (28), awake night $84(13) 1 \mathrm{~min}^{-1} ; \mathrm{p}<0.02$ ) and as a percentage fall in PEF (asleep night $38 \%(6 \%)$, awake night $20 \%(4 \%)$; $<0.01)$.

\section{BRONCHIAL REACTIVITY TO HISTAMINE}

In 11 patients bronchial reactivity to histamine was

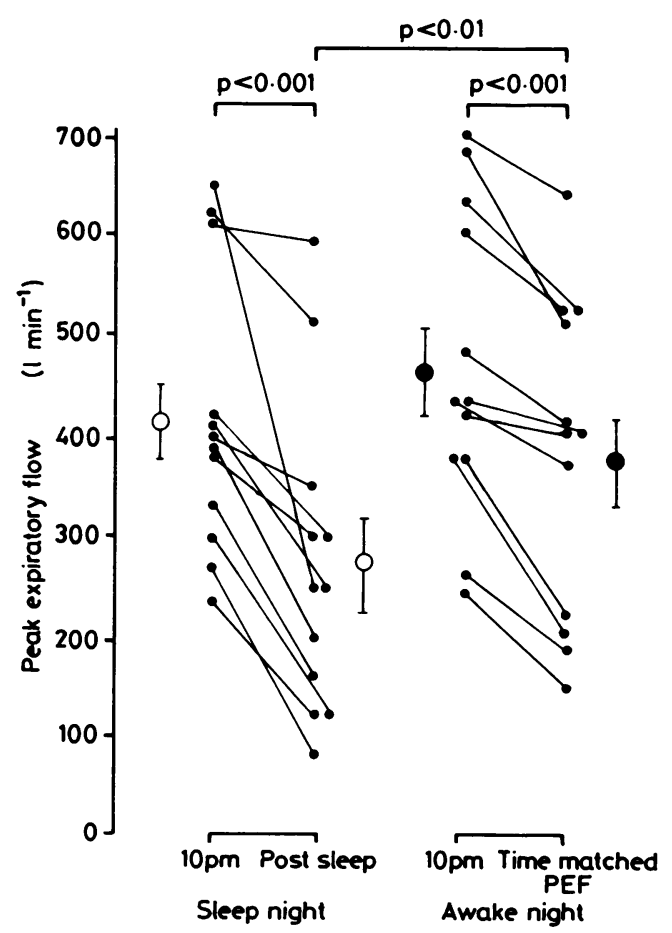

Fig 1 Peak expiratory flow at night and in the morning on both the asleep and the awake nights. All patients developed bronchoconstriction on both nights but, although the $10 \mathrm{pm}$ peak flow rates were not significantly different, morning peak flow was higher after the awake night. 


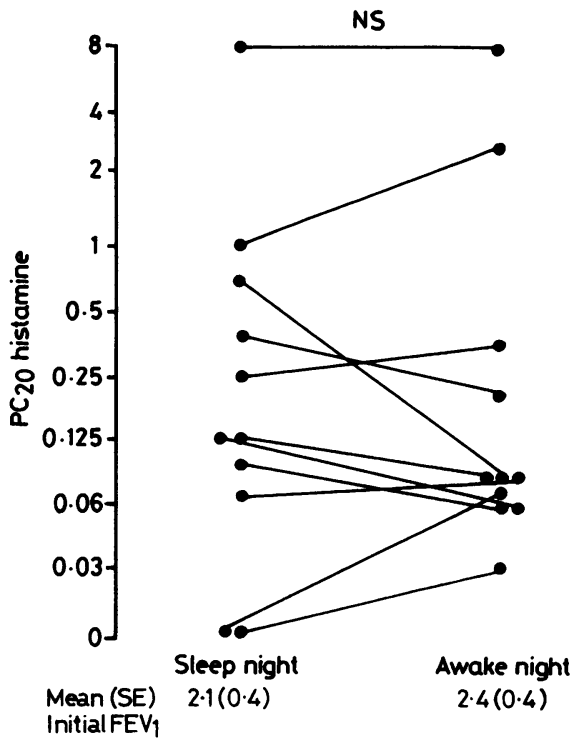

Fig 2 Concentration of histamine required to reduce $F E V_{1}$ by $20 \%\left(P C_{20}\right.$ units) in 11 asthmatic subjects after the asleep night and after the awake night. Overnight wakefulness produced no change in bronchial reactivity to histamine.

assessed at 7.00 after both the asleep night and the awake night. The 12th patient was excluded from histamine challenge because his morning value of $\mathrm{FEV}_{1}$ was less than 1 litre on both mornings. There was no difference between the awake night and the asleep night in the bronchial reactivity to histamine as assessed by the concentration of histamine which reduced $\mathrm{FEV}_{1}$ by $20 \%$ (fig 2 ), even though histamine FEV $_{1}$ was lower after the asleep night.

\section{Discussion}

This study shows that patients with nocturnal asthma develop bronchoconstriction at night even if they are kept awake. Our patients, however, showed greater falls in peak flow on the night when they slept. Furthermore, five patients had to use their bronchodilator aerosols during the asleep night but only two during the awake night, and both of these used the inhaler later on the awake than on the asleep night. Thus the study shows that sleep, while not essential for nocturnal bronchoconstriction, has a role in airway narrowing at night. The data suggest that factors related to sleep account for about half of the overnight fall in peak flow. There was no change in brochial reactivity after overnight wakefulness.

This is the first study of the effect of sleep deprivation on nocturnal asthma to document wakefulness by EEG recording and to exclude the ingestion of caffeine (a bronchodilator), which would con- fuse the results. Our observations therefore extend $\stackrel{\vec{F}}{\stackrel{7}{\longrightarrow}}$ those of Hetzel and Clark, ${ }^{12}$ who attempted to keep $\overrightarrow{0}$ asthmatic subjects awake until the early morning and $\frac{C}{O}$ found that most, but not all, showed their usual $\overline{\frac{5}{3}}$ degree of nocturnal bronchoconstriction before going $\underset{\otimes}{\otimes}$ to sleep. Electroencephalograms, however, were not recorded in their study and their patients may not ${ }^{\circ}$ have stayed awake all night, particularly as they $\vec{\circ}$ "adopted the sleeping posture ... and spent their time $\overrightarrow{\vec{H}}$ reading or listening to the radio." Conflicting evi- $\stackrel{\omega}{\sim}$ dence came from the study of Reinhardt $e t$ al,${ }^{13}$ who found that nocturnal bronchoconstriction in asthmatic children was abolished by sleep deprivation, $\stackrel{ \pm}{\overrightarrow{0}}$ although it is not clear how the children in that study were kept awake.

The finding in the current study that sleep is 0

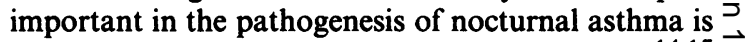
supported by studies in asthmatic shift workers, ${ }^{14}{ }^{17} \overrightarrow{\mathrm{C}}$ in whom the time of sleep determines the time of the $\mathbb{D}$ fall in peak flow, irrespective of the time of day. When $\stackrel{\mathbb{D}}{\vec{D}}$ such workers changed shift their "overnight" 긍 bronchoconstriction became coincident with sleep $\stackrel{\mathbb{D}}{-}$ time by the end of the first sleep period; and both $\vec{\varphi}$ Clark and Hetzel ${ }^{17}$ and Connolly ${ }^{14}$ thought that this change was too rapid to be caused by alterations in circadian variation of hormones, although concentrations of such hormones were not determined.

Why were higher peak flow rates obtained after the $\frac{\partial}{\varnothing}$ awake night? We tried to minimise the differences 0 between the awake and the asleep night but we cannot $\overrightarrow{\vec{O}}$ totally exclude these as factors. Both nights were spent in the same rooms at the same temperature ${ }^{15}$ and precautions were taken to keep the house dust mite count low. ${ }^{16}$ It was not feasible for the patients to be lying down throughout the night they were kept $\frac{\text { 응 }}{7}$ awake, as we would have been unable to keep these $x$ volunteer patients fully awake as defined by the EEG. $\frac{}{3}$ We cannot therefore exclude posture differences as contributory factors in the changes in peak flow. $\frac{\text { }}{3}$ Nocturnal falls in peak flow are, however, unchanged

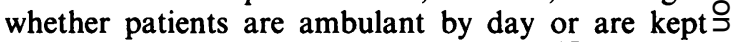
lying in bed throughout the 24 hour day, ${ }^{17}$ and thus $?$ we do not think that posture effects are likely to explain the differences in peak flow.

Although the values of peak flow at $10 \mathrm{pm}$ were no significantly different, there was a trend for PEF to be $\mathcal{N}$ higher at 10 before the awake night. We cannot $\omega$ explain this trend. It was necessary for the patients to know in advance which night they were going to bee awake so that they could arrange work schedules; but $\mathbb{\Phi}$ if they had had a nap (which each denied) before com $\stackrel{?}{+}$ ing in on the awake night the PEF would be expected ${ }^{\circ}$ to have been lower at the start of that night, whereas the trend was towards the reverse. This non- $?$

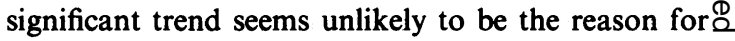


significantly smaller falls in both absolute and percentage PEF on the awake night.

We think it more likely that sleep itself influences nocturnal asthma. Such an effect of sleep could be either direct or indirect. Indirect mechanisms might include the role of sleep in controlling circadian rhythms of hormones, body temperature, ${ }^{15}$ autonomic nervous activity, or other factors. The sleepwake cycle is important in synchronising circadian rhythms, and disruption of this cycle by enforced overnight wakefulness would alter such rhythms. It has been suggested that nocturnal asthma results from overnight falls in circulating adrenaline $\mathrm{e}^{1819}$ and the results of our study could be explained were circadian changes in adrenaline concentrations altered by overnight wakefulness. Previous studies in normal subjects have found no consistent changes in circulating catecholamines during overnight wakefulness, ${ }^{20}$ but clearly measurements are needed in patients with nocturnal asthma. Direct effects of sleep on breathing, such as changes in breathing pattern ${ }^{21}$ and ventilatory control, ${ }^{22}{ }^{23}$ are transient and on arousal return to the patterns found in wakefulness. The effect of sleep on nocturnal asthma is clearly different from these effects as nocturnal bronchoconstriction persists after awakening.

Areas of sleep physiology that merit further exploration as potential causes of nocturnal asthma include REM sleep and vagal tone. Studies in dogs have shown that REM sleep is associated with appreciable variability in airway calibre, which could be abolished by vagal denervation. ${ }^{24}$ We have found that stable asthmatic subjects woken from REM sleep have low expiratory flow rates than when they are woken from other stages of sleep, ${ }^{25}$ suggesting that REM sleep is associated with bronchoconstriction. Thus REM sleep may induce bronchoconstriction that could contribute to the development of nocturnal asthma. Another possible mechanism is suggested by the observation that sleep is associated with increased cardiovascular vagal tone. ${ }^{26}$ If bronchial vagal tone also increased during sleep this could contribute to nocturnal bronchoconstriction.

In 1698 Dr (later Sir) John Floyer, himself an asthmatic, observed, "I have observ'd the Fit always to happen after sleep in the night." 27 Our results confirm that sleep is a factor in the pathogenesis of nocturnal asthma.

We thank $\mathbf{J}$ McFie and M Mackenzie for technical and nursing assistance. This work was supported by the Asthma Research Council.

\section{References}

1 Turner-Warwick M. On observing patterns of airflow obstruction in chronic asthma. $\mathrm{Br} J$ Dis Chest 1977;71:73-86.

2 Montplaisir J, Walsh J, Malo JL. Nocturnal asthma: features of attacks, sleep and breathing patterns. Am Rev Respir Dis 1982;125:18-22.

3 Catterall JR, Douglas NJ, Calverley PMA, et al. Irregular breathing and hypoxaemia during sleep in chronic stable asthma. Lancet 1982;i:301-4.

4 Cochrane GM, Clark TJH. A survey of asthma mortality in patients between ages 35 and 64 in the Greater London hospitals in 1971. Thorax 1975;30:300-5.

5 Hetzel MR, Clark TJH, Branthwaite MA. Asthma: analysis of sudden deaths and ventilatory arrests in hospital. Br Med J 1977;i:808-11.

6 Douglas NJ. Asthma at night. Clinics in Chest Medicine 1985;6:663-74.

7 Anonymous. Asthma at night [Editorial]. Lancet 1983;i:220-2.

8 Rechtschaffen A, Kales A. A manual of standardised terminology, techniques and scoring system for sleep stages of human subjects. Bethesda, Maryland: National Institute of Neurological Disease and Blindness, 1968. (NIH publication No 204.)

9 Becker AB, Keith MD, Simons J, et al. The bronchodilator effects and pharmacokinetics of caffeine in asthma. $N$ Engl $J$ Med 1984;310:743-6.

10 Cockcroft DW, Killian DM, Mellon JJA, Hargreave FE. Bronchial reactivity to inhaled histamine: a method in clinical survey. Clin Allergy 1977;7:235-43.

11 Snedecor GW, Cochrane WG. Statistical methods. 7th ed. Iowa: Iowa State University Press, 1980.

12 Hetzel MR, Clark TJH. Does sleep cause nocturnal asthma? Thorax 1979;34:749-54.

13 Reinhardt D, Schuhmacher P, Fox A, et al. Comparison of the effects of theophylline, prednisolone and sleep withdrawal on airway obstruction and urinary cyclic AMP/cyclic GMP excretion of asthmatic children with and without nocturnal asthma. Int J Clin Pharmacol Ther Tox 1980;18:399-404.

14 Connolly CK. Diurnal rhythms in airway obstruction. Br J Dis Chest 1979;73:357-66.

15 Chen WY, Chai H. Airway cooling and noturnal asthma. Chest 1982;81:675-80.

16 Platts-Mills TAE, Mitchell EB, Nock P, et al. Reduction of bronchial hyper-reactivity during prolonged allergen avoidance. Lancet 1982;ii:675-7.

17 Clark TJH, Hetzel MR. Diurnal variation of asthma. $\mathrm{Br}$ $J$ Dis Chest 1977;71:87-92.

18 Soutar CA, Carruthers M, Pickering CAC. Nocturnal asthma and urinary adrenaline and noradrenaline excretion. Thorax 1977;32:677-83.

19 Barnes P, Fitzgerald G, Brown M, et al. Nocturnal asthma and changes in circulating epinephrine, histamine and cortisol. $N$ Engl J Med 1980;303:263-7.

20 Prinz PN, Vitiello MV, Smallwood RG, Schoene RB, Halter JB. Plasma norepinephrine in normal young and aged men: relationship with sleep. $J$ Gerontol 1984;39:561-7.

21 Douglas NJ, White DP, Pickett CK, et al. Respiration 
during sleep in normal man. Thorax 1982;37:840-4.

22 Douglas NJ, White DP, Weil JV, et al. Hypoxic ventilatory response decreases during sleep in normal men. Am Rev Respir Dis 1982;125:286-9.

23 Douglas NJ, White DP, Weil JV, et al. Hypercapnic ventilatory response in sleeping adults. Am Rev Respir Dis 1982;126:758-62.

24 Sullivan CE, Zamel N, Kozar LF, et al. Regulation of airway smooth muscle tone in sleeping dogs. Am Rev Respir Dis 1979;119:87-98.

25 Shapiro C, Montgomery I, Catterall JR. Breathing bronchoconstriction and sleep stage in nocturnal asthma. [abstract]. Thorax 1982;37:238.

26 Baust $\mathrm{W}$, Bohnert $\mathrm{B}$. The regulation of heart rate during sleep. Exp Brain Res 1969;7:169-80.

27 Floyer J. A treatise of the asthma. London: Wilkin, 1698. 